\title{
Upregulation of pyruvate kinase M2 expression by fatty acid synthase contributes to gemcitabine resistance in pancreatic cancer
}

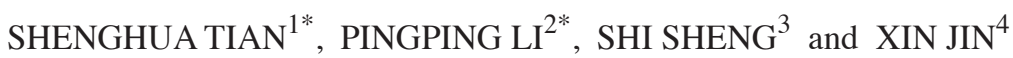 \\ ${ }^{1}$ Department of Endocrinology, Union Hospital, Tongji Medical College, Huazhong University of Science and \\ Technology, Wuhan, Hubei 430022; ${ }^{2}$ Department of Endocrine and Vascular Surgery, Taihe Hospital, Hubei Medical \\ College, Shiyan, Hubei 442000; Departments of ${ }^{3}$ Vascular Surgery and ${ }^{4}$ Digestive Surgical Oncology, Union Hospital, \\ Tongji Medical College, Huazhong University of Science and Technology, Wuhan, Hubei 430022, P.R. China
}

Received May 30, 2016; Accepted March 28, 2017

DOI: $10.3892 / \mathrm{ol} .2017 .7598$

\begin{abstract}
Pancreatic cancer has one of the highest mortality rates of all cancer types. Fatty acid synthase (FASN) is a multifunctional protein homodimer that can convert acetyl coenzyme A (CoA) and malonyl-CoA into palmitate, thus regulating lipogenesis. FASN overexpression has also been shown to cause resistance to gemcitabine, a chemotherapy treatment for pancreatic cancer; however, the mechanism by which this happens is unclear. Analysis of gene expression of FASN and pyruvate kinase M2 (PKM2) in pancreatic cancer was performed using Oncomine microarray gene expression datasets, which demonstrated that FASN and PKM2 were upregulated in pancreatic cancer compared with normal tissue. Specifically, it was demonstrated that FASN enabled the upregulation of PKM2 expression at the mRNA and protein levels, increasing the glucose consumption rate in pancreatic cancer cells. The present study also revealed that decreased levels of FASN reduced resistance to gemcitabine treatment, which was induced by PKM2 overexpression in pancreatic ductal adenocarcinoma cells. Therefore, FASN may represent a novel therapeutic target in pancreatic cancer.
\end{abstract}

\section{Introduction}

Pancreatic cancer has one of the highest mortality rates of all cancer types. It is estimated that $>330,000$ people are diagnosed with pancreatic cancer annually worldwide (1). Despite the relatively low epidemiological ranking of the disease, and although

Correspondence to: Dr Xin Jin, Department of Digestive Surgical Oncology, Union Hospital, Tongji Medical College, Huazhong University of Science and Technology, 1227 Jiefang Road, Wuhan, Hubei 430022, P.R. China

E-mail: jinxinunion@gmail.com

*Contributed equally

Key words: fatty acid synthase, gemcitabine, pyruvate kinase M2, pancreatic cancer, treatment resistance extensive efforts are being made to improve the early diagnosis of the disease, the prognosis of pancreatic cancer remains poor, with a 5-year survival rate of only $4 \%$, making it the fourth-leading cause of all cancer-associated mortality in the United States (2).

The most common type of pancreatic cancer is adenocarcinoma (accounting for $95 \%$ of all cases), which originates from the exocrine region of the pancreas and is classified as pancreatic ductal adenocarcinoma (PDAC) (2). PDAC is insensitive to chemotherapy and radiotherapy, meaning the identification of novel therapeutic targets is imperative (3).

Fatty acid synthase (FASN) is a multifunctional protein homodimer that converts acetyl coenzyme A (CoA) and malonyl-CoA into palmitate, thus regulating lipogenesis (4). Overexpression of FASN has been found to correlate with insulin resistance, type-2 diabetes and pancreatic cancer (5). The overexpression of FASN is an indicator of a poor patient prognosis, a high risk of recurrence and poor survival in numerous cancer types, including cancer of the breast (6), prostate (7), lung (8) and pancreas (9).

FASN overexpression has been shown to cause resistance to gemcitabine-based chemotherapy and radiotherapy in pancreatic cancer patients (10); however, the mechanism by which this happens remains unclear. The Warburg effect is considered to be a possible mechanism for cancer chemoresistance, with the tumor-specific pyruvate kinase M2 (PKM2) protein essential for this effect. It has been reported that the chemoresistance of pancreatic cancer to gemcitabine is PKM2-dependent, and PKM2 is believed to be a therapeutic target of gemcitabine-resistant pancreatic cancer (11). Indeed, the present study found that FASN regulates PKM2 expression and glucose metabolism, leading to gemcitabine chemoresistance in PDAC cells via the direct regulation of PKM2. Collectively, the findings of the present study imply that FASN upregulates PKM2 expression and induces gemcitabine resistance in pancreatic cancer.

\section{Materials and methods}

Oncomine database analysis. mRNA microarray datasets of pancreatic cancer and normal tissue samples were analyzed using the Oncomine microarray gene expression database 
(www.oncomine.org). The parameters maintained including the follow: $\mathrm{P}<0.0001$; fold change $>2$; and the top $10 \%$ of ranked genes.

Plasmids and reagents. Myc-tagged Myc-PKM2 was cloned into a pCMV vector. To construct the Myc-PKM2 plasmid, the full length PKM2 gene was amplified using 293T cells via PCR amplification and cloned into the pCMV-Myc vector (Takara Bio, Inc., Otsu, Japan). Anti-PKM2 (cat. no. 4053; 1:1,000), anti-FASN (cat. no. 3189; 1:1,000) and anti-cleaved caspase-3 (cat. no. 9662; 1:1,000) antibodies were purchased from Cell Signaling Technology, Inc. (Danvers, MA, USA). Anti- $\beta$-Tubulin (cat. no. sc-5274; 1:5,000) was purchased from Santa Cruz Biotechnology, Inc. (Dallas, TX, USA). Gemcitabine was obtained from Eli Lilly (Surrey, UK) and dissolved in distilled water. Pancreatic cancer cells were treated with $10 \mu \mathrm{M}$ gemcitabine and the control group was treated with equal amounts of distilled water for $24 \mathrm{~h}$. Cerulenin was purchased from Sigma-Aldrich (Merck KGaA, Darmstadt, Germany) and $20 \mu \mathrm{M}$ cerulenin was used to treat pancreatic cancer cells for $24 \mathrm{~h}$.

Cell culture and transfection. PDAC cell lines, PANC-1 and MIA PaCa-2, were purchased from the American Type Culture Collection (Manassas, VA, USA) and cultured in 5\% $\mathrm{CO}_{2}$, at $37^{\circ} \mathrm{C}$ and in $95 \%$ humidity. PANC-1 and MIA PaCa-2 cells were cultured in Dulbecco's modified Eagle's medium supplemented with $10 \%$ fetal bovine serum (Thermo Fisher Scientific, Inc., Waltham, MA, USA) and $100 \mathrm{U} / \mathrm{ml}$ penicillin and $100 \mu \mathrm{g} / \mathrm{ml}$ streptomycin (Thermo Fisher Scientific, Inc.). Pancreatic cancer cells with or without FASN knockdown were transfected with Myc-PKM2 plasmids $\left(2 \mu \mathrm{g} / 1 \times 10^{6}\right.$ cells) using Lipofectamine 2000 (Invitrogen; Thermo Fisher Scientific, Inc.) according to the manufacturer's protocol. A total of $24 \mathrm{~h}$ post-transfection, cells were collected for further analyses.

Western blotting. Cells $\left(1 \times 10^{6}\right)$ were lysed with lysis buffer (1\% Nonidet P-40, 1X PBS, 0.1\% sodium dodecyl sulfate and $1 \%$ protease inhibitor cocktail), followed by protein quantification using a bicinchoninic acid (BCA) assay. Samples were diluted in loading buffer containing dithiothreitol and boiled for $5 \mathrm{~min}$. Equal amounts $(50 \mu \mathrm{g})$ of protein for each sample was separated by $10 \%$ SDS-PAGE and transferred onto nitrocellulose membranes. The membranes were immuno-blotted with the aforementioned specific primary antibodies targeted at the protein of interest in $4^{\circ} \mathrm{C}$ overnight. Subsequently, the membrane was wash three times with $1 \mathrm{X}$ TBST and incubated with rabbit IgG (cat. no. MR-R100; 1:3,000) and (mouse IgG; cat.no. MR-M100; 1:3,000) horseradish peroxidase-conjugated secondary antibodies (both Shanghai MRbiotech, Co., Ltd., Shanghai, China) for $1 \mathrm{~h}$ at room temperature, and then visualized using SuperSignal West Pico Stable Peroxide solution (Thermo Fisher Scientific, Inc.).

Reverse transcription-quantitative polymerase chain reaction $(R T-q P C R)$. Total RNA was extracted from cells using TRIzol reagent (Thermo Fisher Scientific, Inc.). The cDNA was synthesized using Superscript II reverse transcriptase (Thermo Fisher Scientific, Inc.). qPCR was performed using IQ SYRB Green Supermix and an iCycleriQTX detection system (Bio-Rad
Laboratories, Inc., Hercules, CA, USA). The following thermocycling conditions were maintained: Denaturing at $95^{\circ} \mathrm{C}$ for $20 \mathrm{sec}$; annealing at $58^{\circ} \mathrm{C}$ for $30 \mathrm{sec}$; and extension at $72^{\circ} \mathrm{C}$ for $30 \mathrm{sec}$ (43 cycles). All signals were normalized against GAPDH and the $2^{-\Delta \Delta \mathrm{Cq}}$ method was used to quantify the fold change (12). The primers used were as follows: FASN forward, 5'-GGTCTTGAGAGATGGCTTGC-3' and reverse, 5'-AAT TGGCAAAGCCGTAGTTG-3'; PKM2 forward, 5'-TCGCAT GCAGCACCTGATT-3' and reverse, 5'-CCTCGAATAGCT GCAAGTGGTA-3'; and GAPDH forward, 5'-ACCCACTCC TCCACCTTTGAC-3' and reverse, 5'-TGTTGCTGTAGC CAAATTCGTT-3'.

RNA interference. Lentivirus-based control and gene-specific short hairpin RNAs (shRNAs) were purchased from Sigma-Aldrich (Merck KGaA). Transfections were performed using Lipofectamine 2000 (Thermo Fisher Scientific, Inc.). A total of $2 \mu \mathrm{g}$ of gene-specific shRNA or shNT (control) were transfected into $293 \mathrm{~T}$ cells $\left(5 \times 10^{5}\right.$ cells $)$. After $48 \mathrm{~h}$ transfection, the cultured medium of $293 \mathrm{~T}$ cells was collected and applied to pancreatic cancer cells. Pancreatic cancer cells were cultured in $5 \% \mathrm{CO}_{2}$, at $37^{\circ} \mathrm{C}$ for $48 \mathrm{~h}$, followed by puromycin $(0.75 \mu \mathrm{g} / \mathrm{ml}$; Sigma-Aldrich; Merck KGaA) selection. Cells were collected $72 \mathrm{~h}$ post-transfection. The knockdown efficiency was confirmed through western blotting or PCR using the aforementioned method. shRNA sequences were as follows: shFASN\#1, CCGGCCTACTGGATGCGTTCTTCAACTCGA GTTGAAGAACGCATCCAGTAGGTTTTTG; shFASN\#2, CCGGGCTGCTAGATGTAGGTGTTAGCTCGAGCTAAC ACCTACATCTAGCAGCTTTTTG; ShPKM2\#1, CCGGCT TTCCTGTGTGTACTCTGTCCTCGAGGACAGAGTACAC ACAGGAAAGTTTTTTG; shPKM2\#2, CCGGGTTCGGAG GTTTGATGAAATCCTCGAGGATTTCATCAAACCTCC GAACTTTTTTG.

Measurements of glucose consumption and lactate production. Culturing medium was collected $24 \mathrm{~h}$ after plasmid transfection or $48 \mathrm{~h}$ after lentivirus infection to allow for the measurement of glucose and lactate concentrations. Glucose levels were determined using a Glucose (GO) assay kit (Sigma-Aldrich; Merck KGaA). Glucose consumption was defined as the difference between the glucose concentration in fresh medium and collected medium. Lactate levels were determined using a Lactate assay kit (Eton Bioscience, Inc., San Diego, CA, USA). The optical densities were measured at a wavelength of $570 \mathrm{~nm}$ in Molecular Devices Spectramax 190 microplate reader (Marshall Scientific, Hampton, NH, USA).

Caspase-3 activity measurement. The activity of caspase-3 was measured using a Caspase-3 Colorimetric Protease assay (Thermo Fisher Scientific, Inc.). Cells were counted and pellets of $3-5 \times 10^{6}$ cells were produced per sample. Cells were lysed using $50 \mu \mathrm{l}$ lysis buffer, followed by protein quantification using a BCA assay. Cytosolic extract was diluted to a concentration of 50-200 $\mu \mathrm{g}$ protein per $50 \mu \mathrm{l}$ in Cell Lysis Buffer (1-4 mg/ml). A total of $50 \mu \mathrm{l}$ of $2 \mathrm{X}$ Reaction Buffer (containing $10 \mathrm{mM}$ DTT) was added to each sample, followed by $5 \mu \mathrm{l}$ of the $4 \mathrm{mM}$ DEVD-pNA substrate $(200 \mu \mathrm{M}$ final concentration), which was then incubated at $37^{\circ} \mathrm{C}$ for $2 \mathrm{~h}$ in the 
A

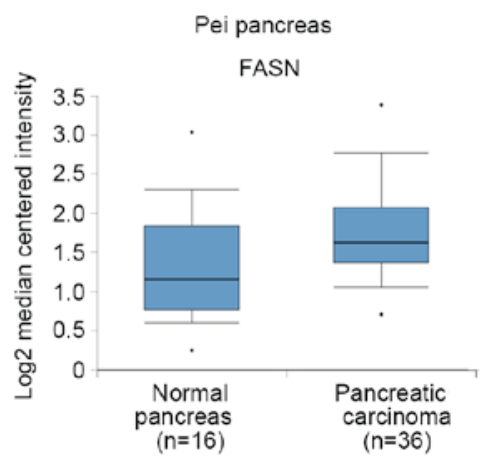

B

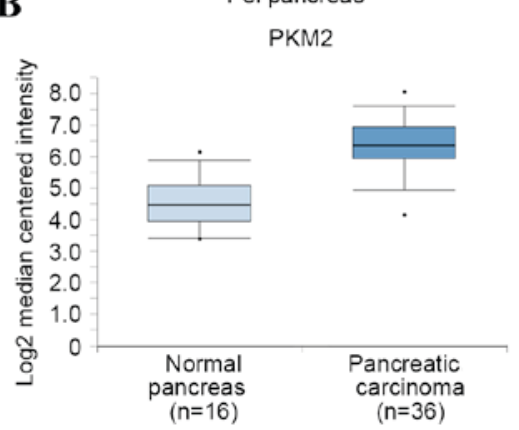

C

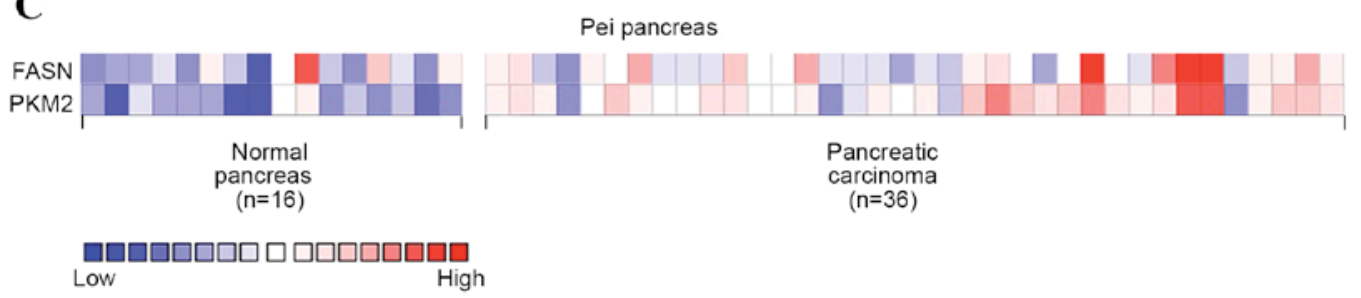

Figure 1. FASN and PKM2 are upregulated in PDAC. (A-C) Elevated (A) FASN, (B) PKM2 expression in PDAC tissue (n=36), compared with healthy pancreatic tissue $(n=16)$. (C) Heat map presenting FASN and PKM2 expression across all samples. Data taken from the Pei pancreas dataset in the Oncomine database.

dark. Reactions were measured using a microplate reader at a wavelength of $405 \mathrm{~nm}$.

Cell proliferation assay. Cell growth was monitored by MTS Cell Proliferation assay according to the manufacturer's instructions (Promega Corporation, Madison, WI, USA). In brief, cells were plated in 96-well plates at a density of 1,000 cells per well. A total $20 \mu \mathrm{l}$ of CellTiter 96R AQueous One Solution Reagent (Promega Corporation) was added to each cell. At $60 \mathrm{~min}$ after incubation (at $37^{\circ} \mathrm{C}$ in a cell incubator), cell proliferation was measured using a microplate reader at a wavelength of $490 \mathrm{~nm}$.

Colony formation assay. Pancreatic cancer cells were seeded into a 6-well plate at a density of $1 \times 10^{3}$ cells/well. Cells were cultured in $5 \% \mathrm{CO}_{2}$ at $37^{\circ} \mathrm{C}$ for 7 days. Cells were fixed with methanol at room temperature for $20 \mathrm{~min}$ and stained with $0.1 \%$ crystal violet at room temperature for $10 \mathrm{~min}$. The number of clones were counted to determine the efficiency of colony formation.

Statistical analysis. Microsoft Excel software (version 2013; Microsoft Corporation, Redmond, WA, USA) was used for statistical analysis. Each experiment was performed in triplicate or with more replicates unless otherwise stated. Unpaired student's t-test was performed for analysis of the statistical significance between groups. A P-value of $<0.05$ was considered to indicate statistical significance.

\section{Results}

FASN and PKM2 are upregulated in PDAC. To investigate the expression of FASN and PKM2 in PDAC, mRNA levels were first analyzed in PDAC and normal pancreas tissues using Oncomine microarray gene expression datasets (www .oncomine.org) $(13,14)$. It was found that FASN and PKM2 were upregulated in PDAC tissues compared with their normal counterparts, using the Pei pancreas dataset in Oncomine (Fig. 1A and B). PKM2 mRNA level was also demonstrated to potentially positively correlate with that of FASN in each sample using the Pei pancreas dataset (Fig. 1C). These results suggest that FASN and PKM2 are upregulated in PDAC, and that PKM2 expression may be positively correlated with that of FASN.

FASN regulates PKM2 expression at the $m R N A$ level. Next, the association between FASN and PKM2 was examined in PDAC. PANC-1 and MIA PaCa-2 cells were treated with non-specific (control) or FASN-specific shRNAs. When FASN was effectively knocked down (Fig. 2A), PKM2 mRNA (Fig. 2B) and protein expression (Fig. 2C) was decreased in these cells (Fig. 2A, C and D). PANC-1 and MIA PaCa-2 cells were then treated with cerulenin, a FASN-specific inhibitor. As expected, the protein level of FASN was decreased in the two cell lines. Notably, the PKM2 protein level in the two cell lines also decreased correspondingly (Fig. 2D). These results indicate that FASN may regulate the expression of PKM2 in PDAC cell lines.

FASN regulates glucose metabolism through PKM2 in PDAC cells. PKM2 is known to promote glucose metabolism in cancer cells (15). To verify the role of FASN in the PKM2-mediated regulation of glucose metabolism in PDAC, PANC-1 and MIA $\mathrm{PaCa}-2$ cells were treated with non-specific or PKM2-specific shRNAs (Fig. 3A). Glucose consumption and lactate production level were significantly decreased in each cell line when PKM2 was knocked down (Fig. 3B and C). PANC-1 and MIA PaCa-2 cells were then treated with non-specific or FASN-specific shRNAs (Fig. 3D). Again, glucose consumption and the lactate production level significantly decreased in 


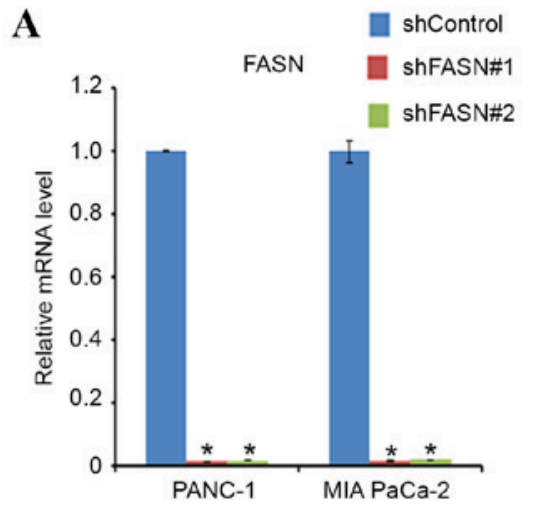

C

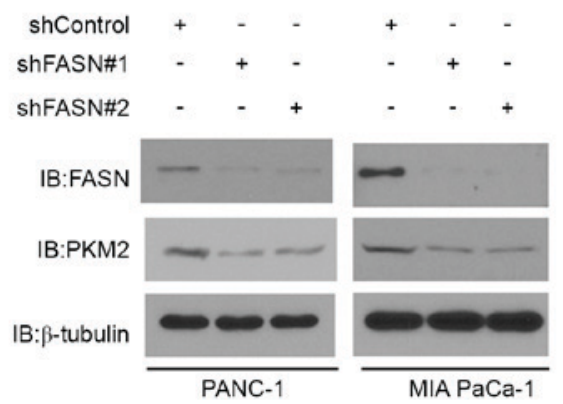

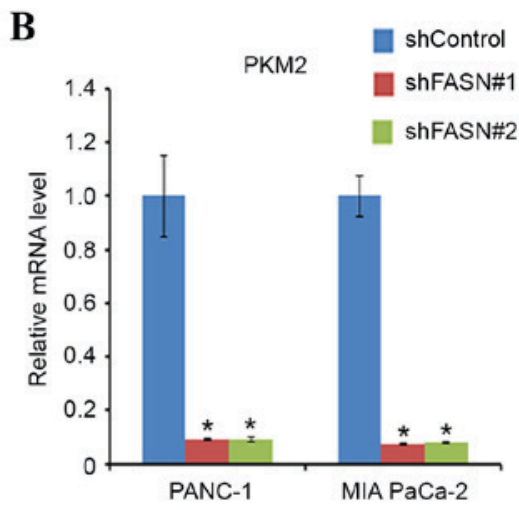

D

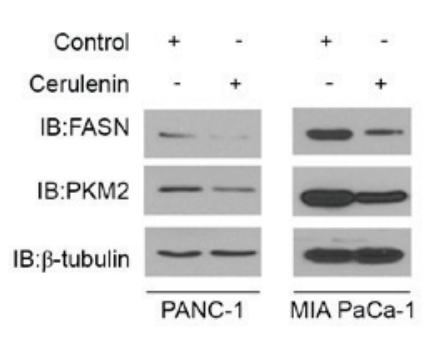

Figure 2. FASN regulates PKM2 expression by increasing mRNA level. (A and B) PANC-1 and MIA PaCa-2 cells were transfected with the indicated shRNAs and after $48 \mathrm{~h}$, cells were harvested for reverse transcription-quantitative polymerase chain reaction. ${ }^{\text {}} \mathrm{P}<0.05 \mathrm{vs}$. shControl. (C) Western blot analysis of WCL obtained from PANC-1 and MIA PaCa-2 cells $48 \mathrm{~h}$ after transfection with indicated shRNAs. $\beta$-tubulin served as an internal control to ensure equal loading. (D) Western blot analysis of WCL obtained from PANC-1 and MIA PaCa-2 cells $48 \mathrm{~h}$ after treatment with the FASN inhibitor cerulenin (20 $\mu \mathrm{M})$. WCL, whole-cell lysate; shRNA, short hairpin RNA; IB, immunoblot.

the cells that underwent FASN-knockdown (Fig. 3E and F). PKM2 overexpression also significantly increased the glucose consumption and lactate production level in the two cell lines; however, FASN-knockdown significantly diminished this tendency (Fig. $3 \mathrm{G}$ and H). These results imply that FASN may regulate glucose metabolism in PDAC cells via PKM2.

FASN induces gemcitabine chemoresistance in PDAC cells via PKM2. Gemcitabine is one of the first-line therapeutic agents for the treatment of pancreatic cancer; however, it has limited efficacy in advanced pancreatic cancer owing to chemoresistance. One possible mechanism of cancer chemoresistance is through the Warburg effect, in which tumor-specific PKM2 may play a pivotal role. Indeed, it has been reported that PKM2 is implicated in gemcitabine chemoresistance (11). In order to investigate the role of FASN in sensitizing PDAC cells to gemcitabine-induced apoptotic death, PDAC cells were treated with gemcitabine alone or in combination with FASN-targeted shRNAs. FASN-knockdown led to an increase in cleaved caspase-3 expression (a pro-apoptotic protein), as well as in caspase-3 activity induced by gemcitabine (Fig. 4A and B). Cell viability was also decreased in FASN-knockdown cells, compared with controls when treated with gemcitabine (Fig. 4C). Consistent with previous findings (10), the present study also indicates that FASN serves a significant role in gemcitabine resistance in PDAC cells.

A PKM2-expressing plasmid was transfected into FASN-knockdown PDAC cells and the evaluation of caspase 3 activity was repeated. It was found that cleaved caspase-3 protein levels and caspase-3 activity were markedly decreased in PKM2-overexpressing PDAC cells (Fig. 5A and B). Cell viability assays were then performed in PKM2-overexpressing, FASN-knockdown or PKM2-overexpression and FASN-knockdown double-treated PANC-1 and MIA PaCa-2 cells in the presence of different concentration of gemcitabine. Higher concentrations of gemcitabine were required to suppress cell growth in PKM2-overexpressing PANC-1 and MIA PaCa-2 cells compared with controls, whereas FASN-knockdown diminished the effects of PKM2 overexpression (Fig. 5C and D). In agreement with this observation, a colony formation assay was performed in the presence of $10 \mu \mathrm{M}$ gemcitabine. In this assay, the colony forming ability of FASN-knockdown PDAC cells was inhibited by gemcitabine, and the effect of gemcitabine resistance induced by PKM2-overexpression was reduced by FASN-knockdown (Fig. 5E). Collectively, these data indicate that inhibition of FASN reduces gemcitabine chemoresistance in PDAC cells, at least in part through the regulation of PKM2.

\section{Discussion}

Pancreatic cancer is a highly aggressive disease, with an incidence rate approximately equal to the mortality rate (16). Surgical resection followed by radiotherapy and/or chemotherapy is currently the first-line treatment; however, $>80 \%$ of patients with pancreatic cancer have unresectable lesions or present with metastatic disease at the time of diagnosis (17). The underlying mechanism for this aggression is unknown; 
A

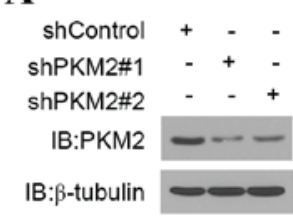

B

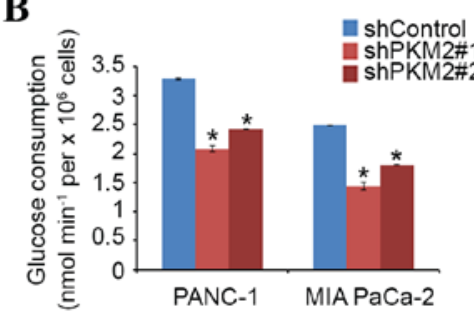

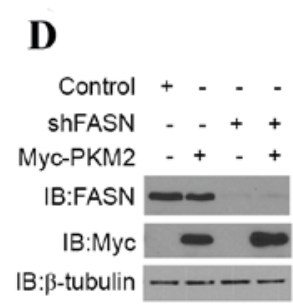

G

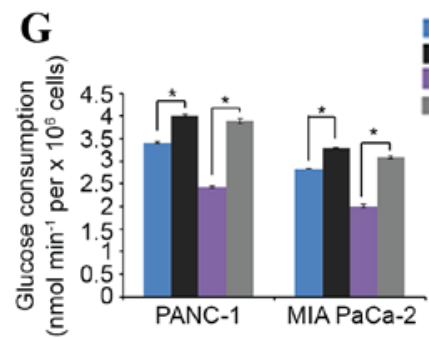

$\mathbf{E}$

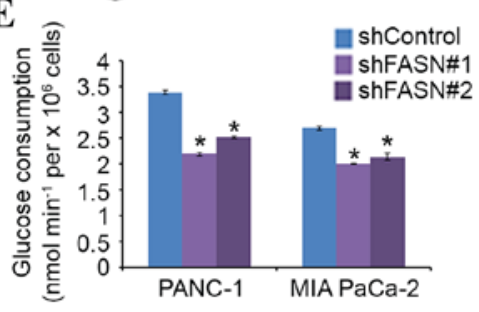

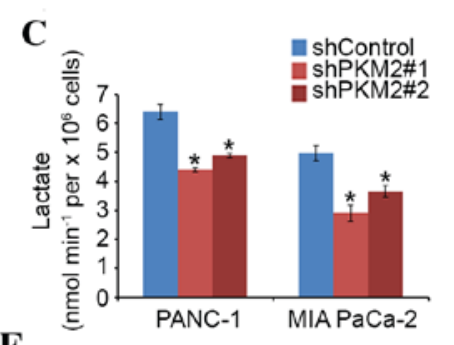

$\mathbf{F}$

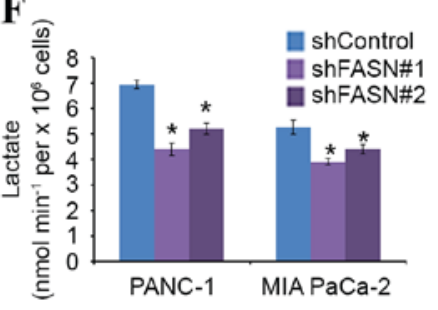

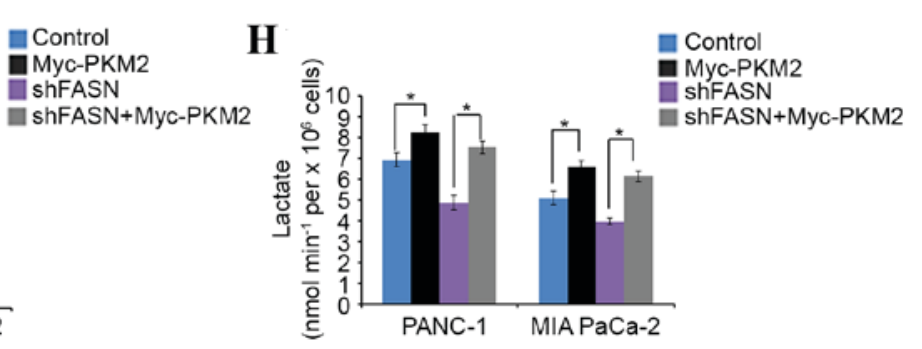

Figure 3. FASN regulates glucose metabolism through PKM2 in PDAC cells. (A-C) PANC-1 and MIA PaCa-2 cells were transfected with PKM2 shRNA and negative control. After $48 \mathrm{~h}$, cells were harvested and (A) western blot analysis of the WCL was performed, as well as measurement of (B) glucose consumption and (C) L-Lactate production in the spent medium. Data are presented as the mean \pm SD from three replicates. (D) PANC-1 cells were transfected with indicated plasmids and, after $24 \mathrm{~h}$, cells were harvested for western blot analysis. (E and F) Measurement of (E) glucose consumption and (F) L-Lactate production in the spent medium of PANC-1 and MIA PaCa-2 cells $48 \mathrm{~h}$ after transfection with FASN shRNA and negative control. Data are presented as the mean \pm SD from three replicates. (G and $\mathrm{H}$ ) Measurement of $(\mathrm{G})$ glucose consumption and $(\mathrm{H}) \mathrm{L}$-Lactate production in the spent medium of PANC-1 and MIA PaCa- 2 cells $48 \mathrm{~h}$ after transfection with the indicated constructs. Data are presented as the mean $\pm \mathrm{SD}$ from three replicates. "P $<0.05$ vs. shControl or shFASN+Myc-PKM2 groups. FASN, fatty acid synthase; PKM2, pyruvate kinase M2; shRNA, short hairpin RNA; SD, standard deviation; IB, immunoblot; Myc, V-myc avian myelocytomatosis viral oncogene homolog.

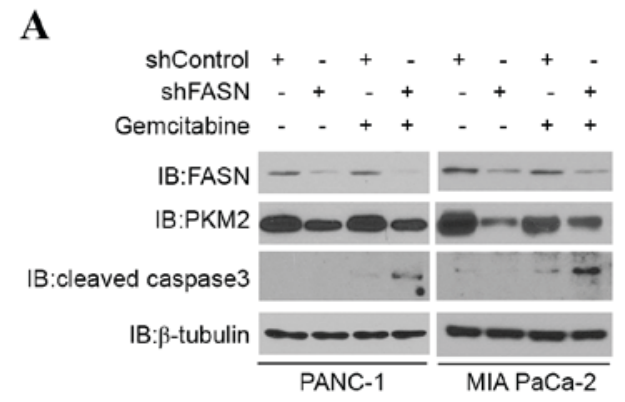

C

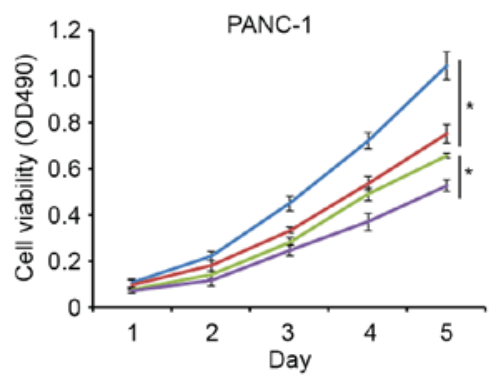

B
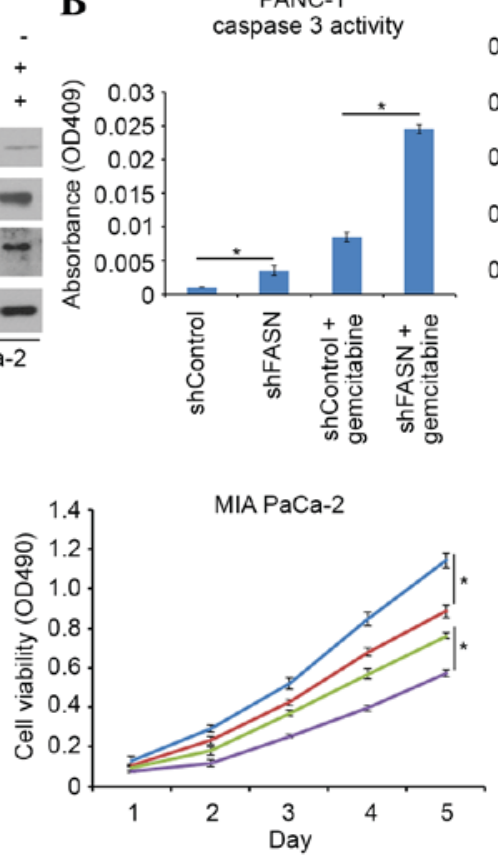

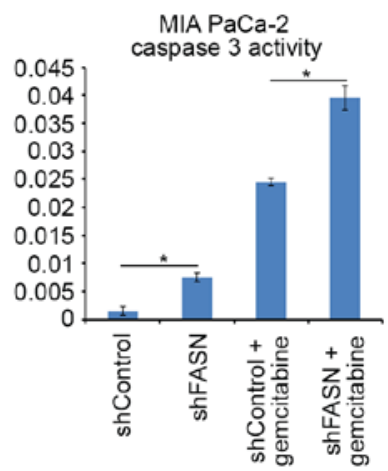

- shControl

- shFASN

- shControl+gemcitabine

- shFASN+gemcitabine

Figure 4. FASN contributes to gemcitabine resistance. (A) Western blot analysis of whole-cell lysates of PANC-1 and MIA PaCa-2 cells $48 \mathrm{~h}$ after transfection with the indicated shRNAs. Cells were treated with or without gemcitabine $(10 \mu \mathrm{M})$ for $24 \mathrm{~h}$ prior to harvest. (B) PANC-1 and MIA PaCa- 2 cells were treated as in (A). At $48 \mathrm{~h}$ after treatment, caspase-3 activity was measured in the cells. Data are presented as the mean \pm SD from two replicates. (C) Cells were treated as in (A). At different time points, cell viability was measured using a MTS Cell Proliferation assay. Data are presented as the mean \pm SD from three replicates. ${ }^{*} \mathrm{P}<0.05$. FASN, fatty acid synthase; SD, standard deviation; OD, optical density; IB, immunoblot. 


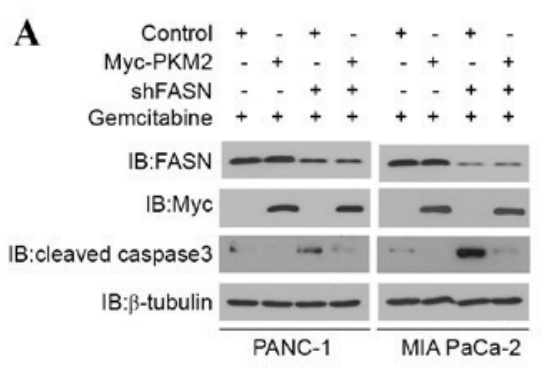

C
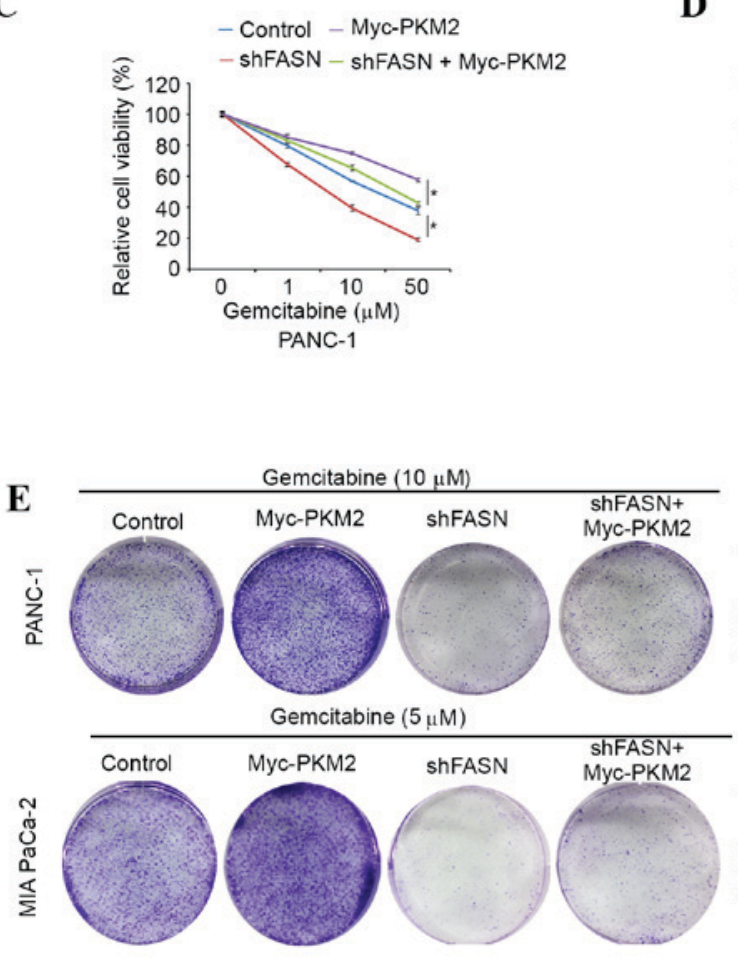
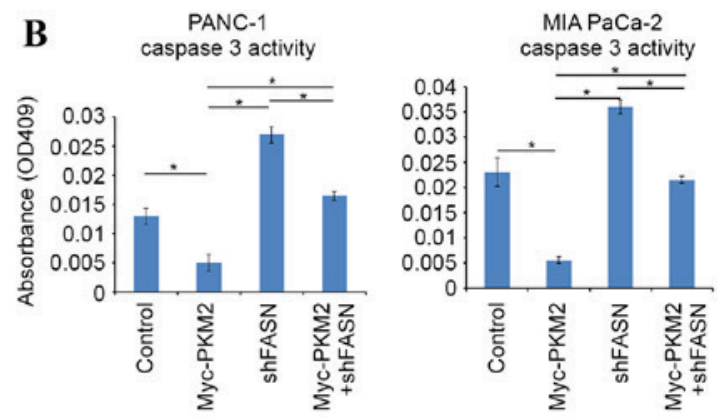

D
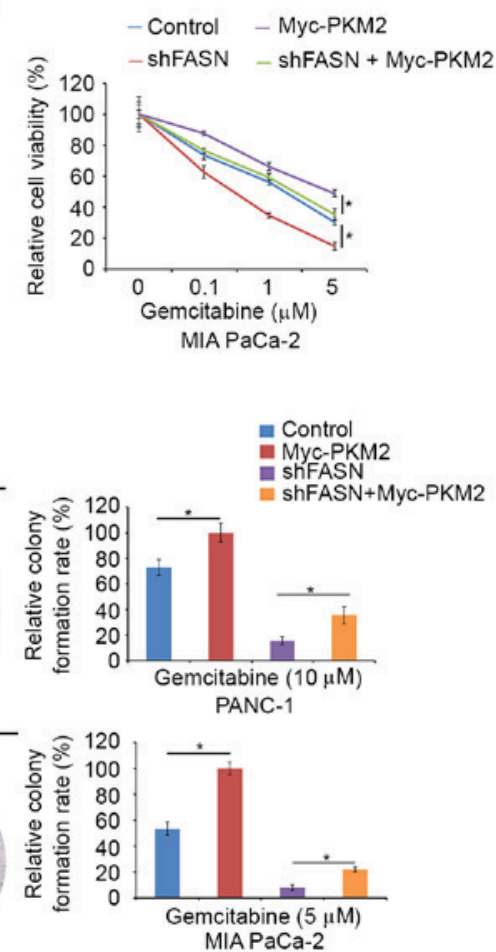

Figure 5. FASN induces gemcitabine chemoresistance in PDAC cells via regulation of PKM2. (A and B) PANC-1 and MIA PaCa-2 cells were transfected with the indicated constructs. Cells were treated with gemcitabine $(10 \mu \mathrm{M})$ for $24 \mathrm{~h}$ prior to harvest. Cells were harvested for (A) western blot analysis and (B) measurement of caspase-3 activity. Data are presented as the mean \pm SD from two replicates. (C and D) PANC-1 and MIA PaCa-2 cells were transfected with the indicated constructs. Cells were treated with different doses of gemcitabine for $24 \mathrm{~h}$ prior to measurement. Cell viability was measured by MTS Cell Proliferation assay. Data are presented as the mean \pm SD from three replicates. ${ }^{*} \mathrm{P}<0.05$. (E) PANC-1 and MIA PaCa-2 cells were transfected with indicated constructs. Cells were treated with or without gemcitabine $(10 \mu \mathrm{M})$. Equal numbers of cells and control cells were seeded onto 60 -mm dishes. After 7 days, the cells were fixed and stained with crystal violet. Data are presented as the mean \pm SD from two replicates. "P $<0.05$. FASN, fatty acid synthase; PDAC, pancreatic ductal adenocarcinoma; PKM2, pyruvate kinase M2; SD, standard deviation; OD, optical density; Myc, V-myc avian myelocytomatosis viral oncogene homolog.

however, alternations in cancer metabolism are believed to contribute to the tumor progress.

On the basis of the Warburg effect, tumor cells have the capacity to produce energy through glycolysis despite lacking a sufficient oxygen supply. Reprogrammed glucose metabolism is one of the hallmarks of cancer (18). Pyruvate kinases are rate-limiting enzymes for aerobic glycolysis in tumor cells, with increased PKM2 expression not only increasing glycolysis but also acting as a nucleus protein kinase to regulate gene expression and promote tumor progression (19). PKM2 functions as an oncogene by promoting cell survival and invasion in PDAC, meaning that it may be a therapeutic target for PDAC (20).

In the current study, FASN and PKM2 were upregulated in pancreatic cancer compared with normal pancreatic tissue, and PKM2 expression was positively correlated with FASN expression in PDAC. The present study has confirmed that
FASN upregulates the mRNA and protein levels of PKM2, and increases the glucose-consumption rate in pancreatic cancer cells. These observations indicate that FASN regulates PKM2 expression and promotes the Warburg effect in pancreatic cancer cells.

FASN, a key lipogenic enzyme, is overexpressed in a number of human cancer types and functions as a metabolic oncogene in pancreatic cancer. FASN has been shown to be consistently upregulated in pancreatic cancer (16). Previous studies have shown that FASN overexpression may contribute to gemcitabine and radiotherapy resistance (10), protect cancer cells against hypoxic conditions and harsh microenvironments (21), and increase the metabolic rate and fatty acid oxidation in cancer cells (22). However, the detailed molecular mechanism by which FASN induces treatment resistance is unclear. 
Pancreatic cancer is a heterogeneous disease and numerous factors contribute to resistance to gemcitabine treatment. One such factor is increased PKM2 expression (11). The experiments described in the present study demonstrated that FASN-knockdown reduced PKM2 expression and alleviated gemcitabine resistance, whereas PKM2-overexpression eliminated the effect induced by FASN-knockdown. These results indicate that in PDAC cells, FASN induces gemcitabine resistance through regulation of PKM2.

The present study demonstrated that FASN upregulated PKM2 expression and glucose metabolism, contributing to gemcitabine resistance in PDAC cells. Therefore, FASN may represent a novel potential therapeutic target for patients with PDAC.

\section{Acknowledgements}

This study was supported by the Scientific Research Training Program for Young Talents of Union Hospital, Tongji Medical College, Huazhong University of Science and Technology.

\section{References}

1. The Lancet Oncology: Pancreatic cancer in the spotlight. Lancet Oncol 15: 241, 2014.

2. Blum R and Kloog Y: Metabolism addiction in pancreatic cancer. Cell Death Dis 5: e1065, 2014.

3. Bian Y, Yu Y, Wang S and Li L: Up-regulation of fatty acid synthase induced by EGFR/ERK activation promotes tumor growth in pancreatic cancer. Biochem Biophys Res Commun 463: 612-617, 2015.

4. Little JL and Kridel SJ: Fatty acid synthase activity in tumor cells. Subcell Biochem 49: 169-194, 2008.

5. Menendez JA, Vazquez-Martin A, Ortega FJ and Fernandez-Real JM: Fatty acid synthase: Association with insulin resistance, type 2 diabetes, and cancer. Clin Chem 55: 425-438, 2009

6. Kuhajda FP, Jenner K, Wood FD, Hennigar RA, Jacobs LB, Dick JD and Pasternack GR: Fatty acid synthesis: A potential selective target for antineoplastic therapy. Proc Natl Acad Sci USA 91: 6379-6383, 1994.

7. Shurbaji MS, Kalbfleisch JH and Thurmond TS: Immunohistochemical detection of a fatty acid synthase (OA-519) as a predictor of progression of prostate cancer. Hum Pathol 27: 917-921, 1996.

8. Piyathilake CJ, Frost AR, Manne U, Bell WC, Weiss H, Heimburger DC and Grizzle WE: The expression of fatty acid synthase (FASE) is an early event in the development and progression of squamous cell carcinoma of the lung. Hum Pathol 31: 1068-1073, 2000.
9. Alo PL, Amini M, Piro F, Pizzuti L, Sebastiani V, Botti C, Murari R, Zotti G and Di Tondo U: Immunohistochemical expression and prognostic significance of fatty acid synthase in pancreatic carcinoma. Anticancer Res 27: 2523-2527, 2007.

10. Yang Y, Liu H, Li Z, Zhao Z, Yip-Schneider M, Fan Q, Schmidt CM, Chiorean EG, Xie J, Cheng L, et al: Role of fatty acid synthase in gemcitabine and radiation resistance of pancreatic cancers. Int J Biochem Mol Biol 2: 89-98, 2011.

11. Kim DJ, Park YS, Kang MG, You YM, Jung Y, Koo H, Kim JA, Kim MJ, Hong SM, Lee KB, et al: Pyruvate kinase isoenzyme M2 is a therapeutic target of gemcitabine-resistant pancreatic cancer cells. Exp Cell Res 336: 119-129, 2015.

12. Livak KJ and Schmittgen TD: Analysis of relative gene expression data using real-time quantitative PCR and the 2(-Delta Delta C(T)) method. Methods 25: 402-408, 2001.

13. Pei H, Li L, Fridley BL, Jenkins GD, Kalari KR, Lingle W, Petersen G, Lou Z and Wang L: FKBP51 affects cancer cell response to chemotherapy by negatively regulating Akt. Cancer Cell 16: 259-266, 2009.

14. Rhodes DR, Yu J, Shanker K, Deshpande N, Varambally R, Ghosh D, Barrette T, Pandey A and Chinnaiyan AM: ONCOMINE: A cancer microarray database and integrated data-mining platform. Neoplasia 6: 1-6, 2004.

15. Lai IL, Chou CC, Lai PT, Fang CS, Shirley LA, Yan R, Mo X, Bloomston M, Kulp SK, Bekaii-Saab T and Chen CS: Targeting the Warburg effect with a novel glucose transporter inhibitor to overcome gemcitabine resistance in pancreatic cancer cells. Carcinogenesis 35: 2203-2213, 2014.

16. Witkiewicz AK, Nguyen KH, Dasgupta A, Kennedy EP, Yeo CJ, Lisanti MP and Brody JR: Co-expression of fatty acid synthase and caveolin-1 in pancreatic ductal adenocarcinoma: Implications for tumor progression and clinical outcome. Cell Cycle 7: 3021-3025, 2008.

17. Zimmon DS and Ferstenberg R: Pancreatic carcinoma. N Engl J Med 326: 1782, 1992.

18. Hanahan D and Weinberg RA: Hallmarks of cancer: The next generation. Cell 144: 646-674, 2011.

19. Wong N, Ojo D, Yan J and Tang D: PKM2 contributes to cancer metabolism. Cancer Lett 356: 184-191, 2015.

20. Li C, Zhao Z, Zhou Z and Liu R: PKM2 Promotes cell survival and invasion under metabolic stress by enhancing warburg effect in pancreatic ductal adenocarcinoma. Dig Dis Sci 61: 767-773, 2016.

21. Furuta E, Pai SK, Zhan R, Bandyopadhyay S, Watabe M, Mo YY, Hirota S, Hosobe S, Tsukada T, Miura K, et al: Fatty acid synthase gene is up-regulated by hypoxia via activation of Akt and sterol regulatory element binding protein-1. Cancer Res 68: 1003-1011, 2008.

22. Legaspi A, Jeevanandam M, Starnes HF Jr and Brennan MF: Whole body lipid and energy metabolism in the cancer patient. Metabolism 36: 958-963, 1987.

(i) $\Theta$ This work is licensed under a Creative Commons Attribution-NonCommercial-NoDerivatives 4.0 International (CC BY-NC-ND 4.0) License. 